\title{
The interaction region of high energy protons
}

\author{
I.M. Dremin \\ Lebedev Physical Institute of RAS, \\ National Research Nuclear University "MEPhI"
}

Content

\author{
1. Introduction \\ 2. Main facts and relations \\ 3. The geometry of the interaction region \\ 4. New tendencies of inelastic interactions \\ 5. Elastic scattering outside the diffraction cone \\ 6. Conclusions \\ References
}

\begin{abstract}
New experimental data about proton-proton collisions obtained at the LHC allow to widen strongly the energy interval where one gets some knowledge about the structure of their interaction region.

Using the unitarity relation in combination with experimental data about the elastic scattering in the diffraction cone, we show how the shape and the darkness of the inelastic interaction region of colliding protons change with increase of their energies. In particular, the collisions become fully absorptive at small impact parameters at LHC energies that results in some special features of inelastic processes. Possible evolution of the shape from the dark core at the LHC to the fully transparent one at higher energies is discussed that implies the terminology of the black disk would be replaced by the black toroid. Another regime could appear with the approach to asymptotics.

The parameter which determines the opacity of central collisions also plays a crucial role in the behavior of the differential cross section of elastic scattering outside the diffraction cone where the predictions of all phenomenological models failed at LHC energies. The role of the ratio of real to imaginary parts of the elastic scattering amplitude at non-forward scattering becomes decisive there as seen from the unitarity condition. The obtained results allow to estimate this ratio outside the diffraction cone for the first time by comparison with experiment
\end{abstract}


at LHC energies, and it happens to be drastically different from its values measured at forward scattering. Moreover, the behaviours of the real and imaginary parts separately differ in different phenomenological models and in the approach based on the unitarity condition. This problem is still waiting for its resolution.

All the conclusions are only obtained in the framework of the indubitable unitarity condition using experimental data about the elastic scattering of protons in the diffraction cone without references to quantum chromodynamics (QCD) or phenomenological approaches.

\section{Introduction}

In this paper, I concentrate on two problems which became of topical interest nowadays in connection to experiments on particle interactions done at the highest available energies of the Large Hadron Collider (LHC) in CERN (Switzerland). To be more definite, our knowledge of the shape and opacity of the interaction region of two colliding protons and the behavior of their elastic scattering amplitude at various transferred momenta will be discussed.

I try to present them in a way most adoptable for newcomers.

The general common approach to both problems considering the irrefutable statement that the total probability of all possible processes must be equal to 1 will be used. It is called the unitarity condition and will be applied to information stemming from experiment about the elastic scattering of protons at small angles within the diffraction cone for the first problem and at larger angles outside it for the second problem. The generality of the approach guarantees the certainty of the obtained results. At the same time, surely, it can not substitute the knowledge of the dynamics of the process but helps get some interesting conclusions about the problems to be approached. That is especially important in view of the limited applications of QCD to quantitative description of experimental data. In addition, some results of the phenomenological models are briefly discussed and confronted to our conclusions as well. The usage in the present paper only these two indubitable ingredients - the unitarity condition and experimental results about the elastic scattering - is decisive for the confidence in derived conclusions.

Why are these problems so important?

The knowledge of the elastic scattering amplitude in the wide range of energies and scattering angles would provide some guides for QCD which is 
practically unapplied yet to this process. Several attempts to use diagrams of elastic scattering of hadrons containing incoherent quarks and gluons were done only in the asymptotical freedom regime of QCD at large transverse momenta with some phenomenological arguments added. Probably, the small angle scattering would ask for account of coherent states of partons in initial protons. The lack of this knowledge prevents further progress in this field. Any guesses from experiment about the behavior of real and imaginary parts of the amplitude are very desirable.

The parton content of hadrons and their spatial interaction region help visualize the collision processes and compare them at different energies. The decisive role is here played by our knowledge of elastic scattering at rather small angles in the diffraction cone. Herefrom we learn about the special regime with the black central core of the interaction region of protons with the total energy in the center of mass system $7 \mathrm{TeV}$ observed at the LHC. In particular, this knowledge helps in developing some models of inelastic processes when their contribution to the unitarity relation is disentangled from elastic terms. Moreover, the further evolution of the spatial region with energy can be speculated so that it leads to some intriguing predictions.

Definite conclusions about transverse momentum dependence (but, unfortunately, not about energy dependence) of the elastic scattering amplitude at larger angles were obtained from the unitarity condition albeit with some adjustable parameters. Fits of experimental data in this region ask for strongly enlarged role of real part of the amplitude compared to the diffraction cone. What concerns phenomenological models, their first attempts to predict the outcome of LHC experiments outside the diffraction cone failed. Their special feature is zero of the imaginary part at the position of the dip of the differential cross section. No such zero is required by the unitarity condition. Till now, there is no definite consensus of these approaches about the behavior of the elastic scattering amplitude there.

\section{Main facts and relations}

Colliding high energy hadrons can either scatter elastically when only two of them appear at the final stage without changing their nature or produce some new particles in inelastic processes. Kinematics of elastic scattering is very simple. It is described by two variables: the squared total energy $s=4 E^{2}$, where $E$ is the energy of one of partners in the center of mass 
system, and the four-momentum transfer squared $-t=2 p^{2}(1-\cos \theta)$ with $\theta$ denoting the scattering angle and $p$ the momentum in the center of mass system. For inelastic processes the kinematics is much more complicated. Therefore, to avoid some complications, it is quite natural to try to get at the first stage some knowledge about the dynamics of the whole process approaching from the analysis of elastic scattering and using such general relation as the unitarity condition. It follows from the irrefutable statement that the total probability of all (elastic + inelastic) processes should be equal to 1 . In this way it relates these two channels of the reaction albeit in rather average integrated form. It is the mainstream of the approach adopted in the present paper.

The experimental data about elastic scattering at a given energy are not very abundant. The only information about this process comes from the measurement of the differential cross section as a function of the transferred momentum at the experimentally available values of $t$ and of the ratio of the real and imaginary parts of the elastic scattering amplitude $f(s, t) \rho(s, t)=$ $\operatorname{Re} f(s, t) / \operatorname{Im} f(s, t)$ just in forward direction $t=0 \rho(s, 0)=\rho_{0}$ but not at any other values of $t$. The latter one is obtained from studies of interference between the nuclear and Coulomb contributions to the amplitude $f$ which becomes practically noticable only in the near-forward direction.

The differential cross section is related to the scattering amplitude $f(s, t)$ in a following way

$$
\frac{d \sigma}{d t}=|f(s, t)|^{2}
$$

Thus, from measurements of the differential cross section at any energy of colliding particles we get the knowledge only about the modulus of the amplitude at the experimentally available values of $t$. The typical shapes of the differential cross section at small and larger values of $|t|$ are demonstrated in Fig. 1 for the LHC energy $\sqrt{s}=7 \mathrm{TeV}$ borrowed from [1, 2].

The most prominent feature of the plots in Fig. 1 is the fast decrease of the differential cross section with increasing transferred momentum $|t|$. As a first approximation at present energies, it can be described at comparatively small transferred momenta in Fig. 1(left) by the exponential shape with the slope $B$ such that

$$
\frac{d \sigma}{d t}=\frac{\sigma_{t}^{2}}{16 \pi} \exp (-B|t|)
$$

where $\sigma_{t}$ denotes the total cross section. This region is called the diffraction peak. The peak becomes higher and its width shrinks with increasing energy 

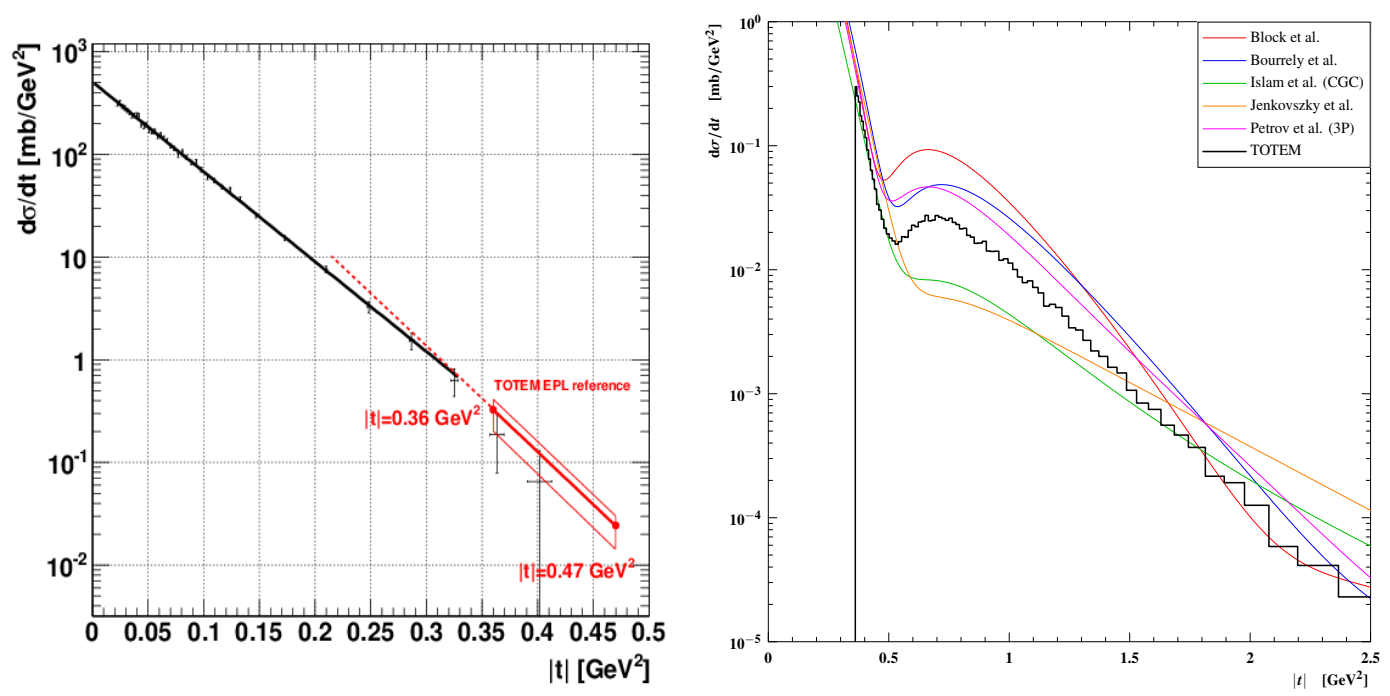

Fig. 1. The differential cross section of elastic proton-proton scattering at the energy $\sqrt{s}=7 \mathrm{TeV}$ measured by the TOTEM collaboration.

Left: The region of the diffraction cone with the $|t|$-exponential decrease [1]. Right: The region beyond the diffraction peak [2]. The predictions of five models are demonstrated.

because both the cross section and the slope increase with energy. The slope slightly depends on $t$ if more careful fits of experimental data are attempted as seen in Fig. 1(left). It diminishes somewhat at energies up to ISR with increasing transferred momentum while starts increasing at the LHC energy $7 \mathrm{TeV}$ (see Fig. 1(left)). Moreover, some oscillations around the exponent were found [3] at the energy $\sqrt{s} \approx 11 \mathrm{GeV}$. The review of the early data can be found in [4]. Interesting by themselves, these details will be not very important further for our approach because the integrals of (2) will be used where they can be accounted by slight variation of $B$. In fact, the values of $B$ at small $|t|<0.3 \mathrm{GeV}^{2}$ are important.

At larger values of $|t|$ outside the peak we observe the dip and slower decrease of the plots with $|t|$ compared to the diffraction cone (see Fig. 1(right)). Let us note that the normalization of the amplitude is fixed by Eqs (1), (2).

To disentangle the real and imaginary parts of the amplitude from the ratio $\rho$, one needs the help from theorists. From the theoretical side, the most reliable information comes from the unitarity condition. The unitarity of the 
$S$-matrix $S S^{+}=1$ imposes definite requirements on the amplitude of elastic scattering $f(s, t)$ and amplitudes of inelastic processes $M_{i}$. In the $s$-channel it looks [5, 6] like

$$
\frac{s}{8 \pi^{3 / 2}} \iint d \theta_{1} d \theta_{2} \frac{\operatorname{Im} f(p, \theta)=I_{2}(p, \theta)+g(p, \theta)=}{\sqrt{\left[\sin \theta_{1} \sin \theta_{2} f\left(p, \theta_{1}\right) f^{*}\left(p, \theta_{2}\right)\right.}}+g(p, \theta) .
$$

The region of integration in (3) is given by the conditions

$$
\left|\theta_{1}-\theta_{2}\right| \leq \theta, \quad \theta \leq \theta_{1}+\theta_{2} \leq 2 \pi-\theta .
$$

The non-linear integral term represents the two-particle intermediate states of the incoming particles. The function

$$
g(p, \theta) \propto \sum_{i} \int d \Phi_{i} M_{i} M_{i}^{*}(\theta)
$$

represents the shadowing contribution of the inelastic processes to the imaginary part of the elastic scattering amplitude. Following [7] it is called the overlap function. This terminology is ascribed to it because the integral there defines the overlap within the corresponding phase space $d \Phi_{i}$ between the matrix element $M_{i}$ of the $i$-th inelastic channel and its conjugated counterpart with the collision axis of initial particles turned by the angle $\theta$ of proton scattering in the elastic process. It is positive at $\theta=0$ but can change sign at $\theta \neq 0$ due to the relative phases of inelastic matrix elements $M_{i}$ 's.

At $t=0$ it leads to the optical theorem

$$
\operatorname{Im} f(s, 0)=\sigma_{t} / 4 \sqrt{\pi}
$$

and to the general statement that the total cross section is the sum of cross sections of elastic and inelastic processes

$$
\sigma_{t}=\sigma_{e l}+\sigma_{i n}
$$

i.e., that the total probability of all processes is equal to one.

That allows to estimate the real and imaginary parts separately just in forward direction $t=0$ after the values of $\rho_{0}$ and $\sigma_{t}$ are measured.

The real and imaginary parts of the amplitude are, in general, related at any $t$ by the dispersion relations as parts of a single analytic function. 
This approach was used for predictions of the energy behavior of $\rho_{0}$ and was only successful at the qualitative level because its accuracy depends on extrapolations of $\operatorname{Im} f(s, 0)$ and, consequently, of $\sigma_{t}$ to higher energies. The similar treatment at the arbitrary values of $t$ asks for some additional assumptions, and the conclusions strongly depend on them.

The theoretical approaches differ in ascribing different roles for the relative contributions of real and imaginary parts at $t \neq 0$. Unfortunately, the available tools are rather moderate and can not exploit the power of QCD at full strength. Elastic scattering implies that the same hadrons are observed in the final state. It means that the partons inside them acted collectively while the QCD methods are applicable to incoherent interactions of individual partons at high transferred momenta. Therefore, the phenomenological models and some insights from the unitarity relation are mostly used.

We also know from experiment the energy behavior of real and imaginary parts of the amplitude (or their ratio $\rho(s, 0)=\rho_{0}$ ) in forward direction $t=0$. At high energies this ratio is rather small. For proton-proton scattering, it is negative at lower energies (reaching the values about -0.3), becomes equal to zero at energies about $100 \mathrm{GeV}$, exhibits a positive maximum and diminishes to about 0.1 at LHC energy $7 \mathrm{TeV}$. Most phenomenological models aim to fit experimental data about the differential cross section and the ratio $\rho(s, 0)$ in a wide energy range and to predict them at higher energies. Up to now, we can not claim that the desired aim has been achieved as seen, in particular, in Fig. 1(right) where the failure of predictions of five theoretical models at 7 $\mathrm{TeV}$ is shown even though all of them were quite successful at lower energies.

This situation is described in more details in the review paper [6]. I will not repeat it here. Instead, I concentrate on the described above two important problems.

\section{The geometry of the interaction region}

The structure of protons is one of the main problems in particle physics. It is well known [8] that up to now there exists the $\approx 7 \sigma$ disagreement between the proton charge radius determined from muonic hydrogen and from electron-proton systems: atomic hydrogen and ep elastic scattering. In deep inelastic electron-proton collisions the partonic structure of protons is successfully studied. The point-like nature is ascribed to the colliding electron. Therefore, the interaction region is defined by the proton size. Its 
size and opacity (or darkness) are determined by the Fourier-image of the generalized parton distribution functions of protons depending on the total energy and the virtuality of the exchanged photon measured in experiment. Both the size and the opacity evolve with energy because the parton content of the proton evolves.

In proton-proton as well as in proton-nucleus and nucleus-nucleus collisions, both objects possess some complicated internal structure. The partons of one of them can interact with many partons from another one distributed somehow within some space volume. Moreover, there can be coherent interactions of some groups of partons. Therefore, it is hard to disentangle the individual contributions. The correlation femtoscopy, using its correspondence to the well known in astrophysics Hanbury-Brown and Twiss intensity interferometry, is widely applied for studies of the space-time structure in inelastic processes. The correlations between the momenta of newly created particles (mostly, pions) reveal the space structure of the interaction region. This technique is especially successful in applications to nuclei but meets some problems [9] for smaller objects like protons connected with the Heisenberg uncertainty relation. The uncertainty limit is about $1 \mathrm{fm}$ for the current high energy experiments. The coherence of individual sources should be taken into account for such systems.

Here, we show that it is possible to study the space structure of the interaction region of colliding protons even at smaller distances using the information about their elastic scattering. The transverse size of this region will be discussed. We do not consider the longitudinal and time sizes because they are strongly related to the model dependent suppositions of the partonic structure of protons (the relative contributions of partons with definite shares of the longitudinal momentum). In fact, the role of the generalized parton distribution functions integrated over the longitudinal momenta is to be studied. Experimental results about properties of the diffraction cone, which automatically account for nonperturbative dynamics of the process and coherence of unknown internal sources, define main features of the transverse structure. The parameters obtained from experimental data about elastic processes are directly related to such properties of this region as its transverse size and opacity (or blackness). Their energy dependence determines its evolution with collision energy.

To define the geometry of the collision we must express all characteristics presented by the angle $\theta$ and the transferred momentum $t$ in terms of the transverse distance between the centers of the colliding protons called the 
impact parameter $b$. It is easily done by the Fourier - Bessel transform of the amplitude $f$ which retranslates the momentum data to the transverse space features and is written as

$$
i \Gamma(s, b)=\frac{1}{2 \sqrt{\pi}} \int_{0}^{\infty} d|t| f(s, t) J_{0}(b \sqrt{|t|}) .
$$

The unitarity condition in the $b$-representation reads

$$
G(s, b)=2 \operatorname{Re} \Gamma(s, b)-|\Gamma(s, b)|^{2} .
$$

The left-hand side (the overlap function in $b$-representation) describes the transverse impact-parameter profile of inelastic collisions of protons. It is just the Fourier - Bessel transform of the overlap function $g$. It satisfies the inequalities $0 \leq G(s, b) \leq 1$ and determines how absorptive is the interaction region depending on the impact parameter (with $G=1$ for the full absorption and $G=0$ for the complete transparency). The profile of elastic processes is determined by the subtrahend in Eq. (9). If $G(s, b)$ is integrated over the impact parameter, it leads to the cross section of inelastic processes. The terms on the right-hand side would produce the total cross section and the elastic cross section, correspondingly, as it should be according to Eq. (7). The overlap function is often shown in relation with the opacity (or the eikonal phase) $\Omega(s, b)$ such that $G(s, b)=1-\exp (-\Omega(s, b))$. Thus, the full absorption corresponds to $\Omega=\infty$ and the complete transparency to $\Omega=0$.

Even though the impact parameter can not be directly measured, the geometric picture is instructive and closely related to such experimentally found characteristics as the ratio of the diffraction cone slope to the total cross section that provides immediate guides to its energy evolution. The impact parameter profiles of elastic and inelastic hadron collisions are derived as Fourier - Bessel transforms of the measurable data. They help us visualize the geometrical picture of partonic interactions indicating their space extension and the intensity. Our intuitive guesses about the space-time development of these processes can be corrected in this way.

The diffraction cone contributes mostly to the Fourier - Bessel transform of the amplitude. Using the above formulae, one can write the dimensionless $\Gamma$ as

$$
i \Gamma(s, b)=\frac{\sigma_{t}}{8 \pi} \int_{0}^{\infty} d|t| \exp (-B|t| / 2)(i+\rho(s, t)) J_{0}(b \sqrt{|t|}) .
$$


Here, the diffraction cone approximation (2) is inserted. Herefrom, one calculates

$$
\operatorname{Re} \Gamma(s, b)=\frac{1}{Z} \exp \left(-\frac{b^{2}}{2 B}\right),
$$

where we introduce the dimensionless ratio of the cone slope (or the elastic cross section) to the total cross section

$$
Z=\frac{4 \pi B}{\sigma_{t}}=\frac{\sigma_{t}}{4 \sigma_{e l}}
$$

This dependence on the impact parameter was used, in particular, in [10]. Possible small deviations from the exponential behavior (see, e.g., [3, 4]) inside the cone do not practically change the value of the integral contribution. The differential cross section is quite small outside the diffraction peak and also does not influence the impact parameter profile $G$. Therefore, our task happens to be practically independent of the second problem.

As was mentioned, the ratio $\rho(s, t)$ is very small at $t=0$ and, at the beginning, we neglect it and get

$$
G(s, b)=\frac{2}{Z} \exp \left(-\frac{b^{2}}{2 B}\right)-\frac{1}{Z^{2}} \exp \left(-\frac{b^{2}}{B}\right) .
$$

For central collisions with $b=0$ it gives

$$
G(s, b=0)=\frac{2 Z-1}{Z^{2}} .
$$

This formula is very important because it follows herefrom that the darkness at the very center is fully determined by the parameter $Z$, i.e. by the ratio of experimentally measured characteristics - the width of the diffraction cone $B$ (or $\sigma_{e l}$ ) to the total cross section. Their energy evolution defines the evolution of the absorption value. The interaction region becomes completely absorptive $G(s, 0)=1$ in the center only at $Z=1$ and the absorption diminishes for other values of $Z$.

In the Table, we show the energy evolution of $Z$ and $G(s, 0)$ for $p p$ and $p \bar{p}$ scattering as calculated from experimental data about the total cross section and the diffraction cone slope at corresponding energies. Let us point out that starting from ISR energies the value of $Z$ decreases systematically and at LHC energies becomes equal to 1 within the accuracy of measurements of $B$ and $\sigma_{t}$. 
Table. The energy behavior of $Z$ and $G(s, 0)$.

\begin{tabular}{|l|l|l|l|l|l|l|l|l|l|}
\hline$\sqrt{s}, \mathrm{GeV}$ & 2.70 & 4.11 & 4.74 & 7.62 & 13.8 & 62.5 & 546 & 1800 & 7000 \\
\hline$Z$ & 0.64 & 1.02 & 1.09 & 1.34 & 1.45 & 1.50 & 1.20 & 1.08 & 1.00 \\
$G(s, 0)$ & 0.68 & 1.00 & 0.993 & 0.94 & 0.904 & 0.89 & 0.97 & 0.995 & 1.00 \\
\hline
\end{tabular}

The impact parameter distribution of $G(s, b) \sqrt{13})$ has the maximum at $b_{m}^{2}=-2 B \ln Z$ with full absorption $G\left(b_{m}\right)=1$. Its position depends both on $B$ and $Z$.

Note, that, for $Z>1$, one gets the incomplete absorption $G(s, b)<$ 1 at any physical $b \geq 0$ with the largest value reached at $b=0$ because the maximum appears at non-physical values of $b<0$. The disk is semitransparent.

At $Z=1$, the maximum is positioned exactly at $b=0$, and the absorption is absolutely strong there $G(s, 0)=1$. The disk center becomes impenetrable (black).

At $Z<1$, the maximum shifts to positive physical impact parameters. The dip is formed at the center that leads to the concave shape of the inelastic interaction region approaching the toroid shape. It becomes deeper at smaller $Z$. The limiting value $Z=0.5$ leading to the complete transparency at the center $b=0$ is considered in more details below.

The maximum absorption in central collisions $G(s, 0)=1$ is reached at the critical point $Z=1$ which is the case at the LHC energy $\sqrt{s}=7$ $\mathrm{TeV}$ as seen from the Table. Therefore it is considered first. Moreover, the strongly absorptive core of the interaction region grows in size as we see from expansion of Eq. (13) at small impact parameters:

$$
G(s, b)=\frac{1}{Z^{2}}\left[2 Z-1-\frac{b^{2}}{B}(Z-1)-\frac{b^{4}}{4 B^{2}}(2-Z)\right] .
$$

The second term proportional to $b^{2}$ vanishes at $Z=1$, and $G(b)$ develops a plateau which extends to quite large values of $b$ about $0.4-0.5 \mathrm{fm}$. The plateau is very flat because the third term starts to play any role at $7 \mathrm{TeV}$ (where $B \approx 20 \mathrm{GeV}^{-2}$ ) only at even larger values of $b$. The structure of the interaction region with a central core at energies $7-8 \mathrm{TeV}$ is also supported (see Fig. 2, where it is compared with corresponding structures at ISR energies) by direct computation [11] using the experimental data of the TOTEM collaboration [1, 2] about the differential cross section in the region of $|t| \leq 2.5 \mathrm{GeV}^{2}$. 


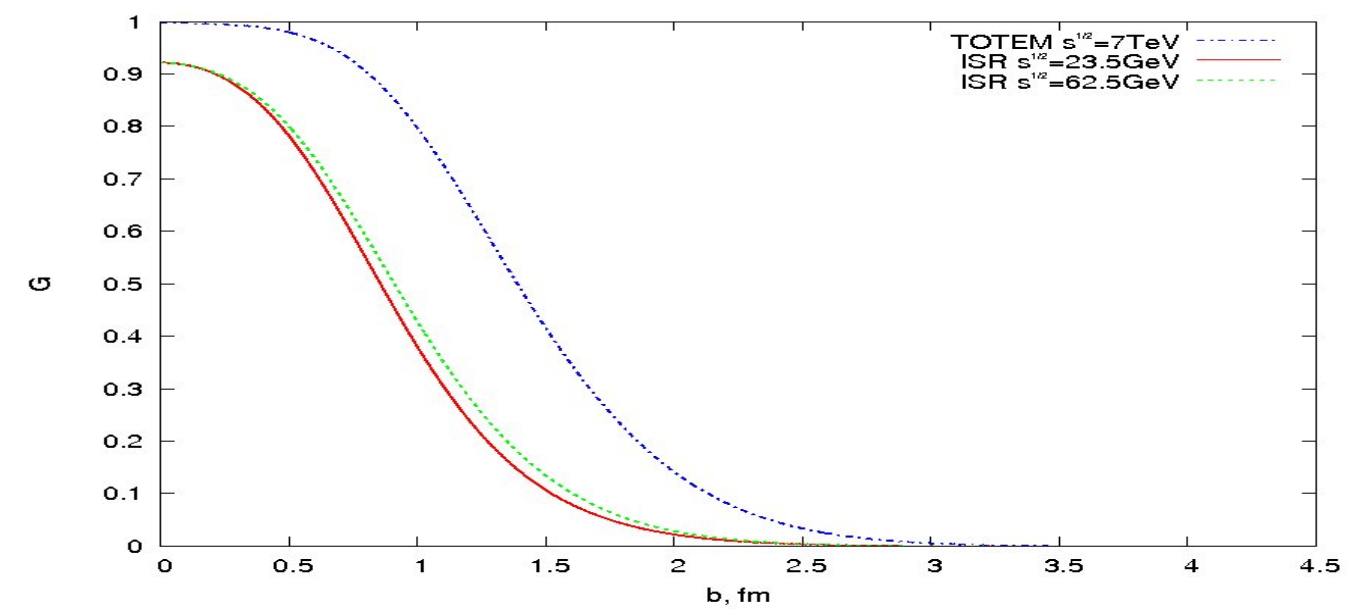

Fig. 2. The overlap function $G(s, b)$ at $7 \mathrm{TeV}$ (upper curve) [11] compared to those at ISR energies $23.5 \mathrm{GeV}$ and $62.5 \mathrm{GeV}$ (all of them are computed by using the fit of experimental data according to the phenomenological model [12]).

The results of analytical calculations according to (13) and the direct computation practically coincide (see Fig. 1 in [13]). It was also shown in [13] that this two-component structure with the central black core and more transparent periphery is well fitted by the expression with the abrupt (Heaviside-like) change of the exponential. However, it is still pretty far from the black disk limit because the peripheral region at $b$ near $1 \mathrm{fm}$ is very active and shows strong increase compared to ISR energies [11. This is demonstrated in Fig. 3 where the difference $\Delta G(b)=G\left(s_{1}, b\right)-G\left(s_{2}, b\right)$ between the overlap functions at different energies $s_{1}$ and $s_{2}$ is displayed.

The lower plot in Fig. 3, obtained in [12], demonstrates that even within the quite narrow interval of ISR energies the role of peripheral interactions with the impact parameters about $1 \mathrm{fm}$ increases in inelastic processes with energy increase. Even more spectacular is the peripheral increase when one comes from ISR to LHC energies as seen in the upper plot in Fig. 3. Moreover, the darkness of the central core strongly increases in Figs 2 and 3 that becomes especially important as we discuss in detail in the next section.

It is interesting that the positivity of $G(s, b)$, i.e. of $\sigma_{\text {inel }}(s, b)$, imposes some limits on the relative role of $B$ and $\sigma_{t}$. Namely, it follows from Eq. (14) 


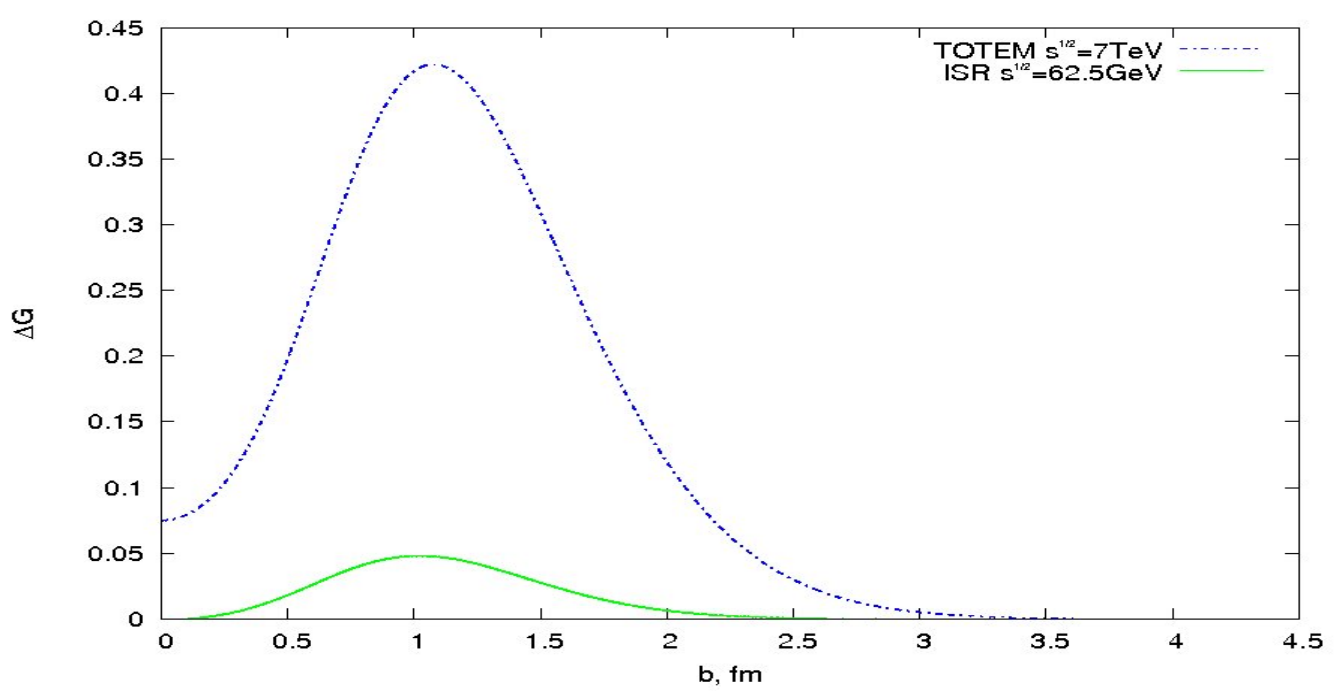

Fig. 3. The difference between the overlap functions at different energies [11, 12. Dash-dotted curve is for $7 \mathrm{TeV}$ and $23.5 \mathrm{GeV}$ energies, solid curve is for $62.5 \mathrm{GeV}$ and $23.5 \mathrm{GeV}$.

Conclusion: The parton density at the periphery strongly increases!

that

$$
2 Z=\frac{8 \pi B}{\sigma_{t}}=\frac{\sigma_{t}}{2 \sigma_{e l}} \geq 1
$$

This relation implies that the slope $B$ should increase with energy at least as strong as the total cross section $\sigma_{t}$.

This inequality is fulfilled at present and intermediate energies. If the value of $Z$ will decrease at energies above $7 \mathrm{TeV}$, as one could expect from its tendency shown in the Table, and approach $Z=0.5$ this inequality can be saturated. We discuss first what happens in the region $0.5 \leq Z \leq 1$. The values $Z<0.5$ will be discussed at the end of this section.

It is usually stated that the equality $2 Z=8 \pi B / \sigma_{t}=\sigma_{t} / 2 \sigma_{e l}=1$ corresponds to the black disk limit. Surely, the equality of elastic and inelastic cross sections is fulfilled $\sigma_{e l}=\sigma_{i n}=0.5 \sigma_{t}$. However, beside this equality, the scattering on the black disk should result in the special non-exponential shape of the differential cross section of the type

$$
\frac{d \sigma}{d t} \propto \frac{J_{1}^{2}(R \sqrt{|t|})}{|t|}
$$


It possesses a zero at $|t| \approx 3.67 / B$ if the relation $R^{2}=4 B$ is used. At the energy $7 \mathrm{TeV}$ this zero should be placed at $|t|=0.2 \mathrm{GeV}^{2}$. That is not confirmed by experiment as well as the equality of cross sections of elastic and inelastic collisions.

In principle, one can not exclude possible fast change of the regime of the exponential decrease in the diffraction cone (2) by a new one. The appearance of another, more steep exponent at the end of the diffraction cone in Fig. 1(left) at $7 \mathrm{TeV}$ and the noticable shift of the dip position to smaller transferred momenta at LHC compared to lower energies could be the first signs of it.

Nevertheless, we continue to study the situation assuming that the exponential regime valid up to LHC will persist at higher energies. One sees from Eq. (14) that $G(s, b=0)=0$ at $Z=0.5$, i.e. the inelastic interaction region is completely transparent in central collisions. Surely, one should not call it as a black disk. This paradox is resolved [14] if we write the inelastic profile of the interaction region using Eq. (13). At $Z=0.5$ it looks like

$$
G(s, b)=4\left[\exp \left(-\frac{b^{2}}{2 B}\right)-\exp \left(-\frac{b^{2}}{B}\right)\right]
$$

We see that one must rename the black disk as a black toroid (or a black ring if we consider its two-dimensional projection) with full absorption $G\left(s, b_{m}\right)=1$ at the impact parameter $b_{m}=R \sqrt{0.5 \ln 2} \approx 0.59 R$, complete transparency at $b=0$ and rather large half-width about $0.7 R$. Thus, the evolution to values of $Z$ smaller than 1 at higher energies (this can happen if the decreasing tendency of $Z$ with energy shown in the Table persists) would imply quite special transition from the critical two-scale regime at the LHC to the concave toroid-like (or ring-like in two dimensions) configurations of the interaction region if the exponential shape of the diffraction cone, described by Eq. (2), persists.

It looks as if the protons penetrate through one another at central collisions, just scattering elastically, while peripheral collisions become responsible for inelastic processes. Elastic and inelastic profiles get equal only at $b=b_{m}$. Elastic one dominates at $b<b_{m}$ while inelastic one at periphery $b>b_{m}$.

Is the parton coherence inside each colliding proton responsible for that? Can we observe its effects similar to difference of light scattering in the water (coherence!) and in the air (decoherence and fluctuations are in charge of the blue color of the sky!)? Elastic and inelastic profiles get equal only at 


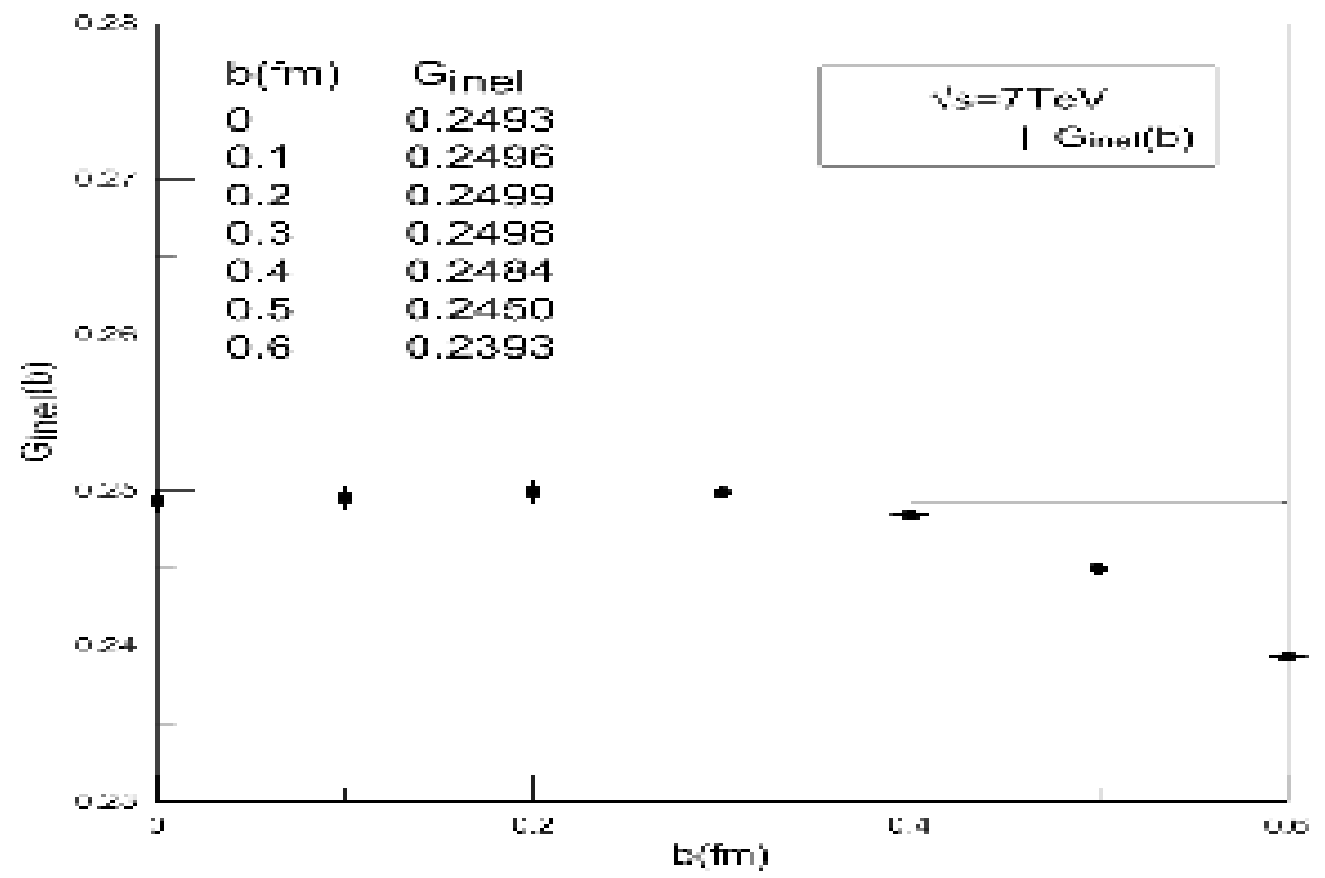

Fig. 4. The impact parameter dependence of the function $G_{\text {inel }}(b)=$ $0.25 G(b)$ at $7 \mathrm{TeV}$ [15. It is obtained using the fit of experimental data according to the model [12]. 
$b=b_{m}$. Elastic one dominates at $b<b_{m}$ while inelastic one at periphery $b>b_{m}$.

Paradoxically enough, it pushes us back to the early suggestions that inelastic processes are more peripheral (recall the one-pion exchange model!) than elastic scattering which is a shadow of inelastic interactions (more pions exchanged). This tendency is clearly seen already at present energies as demonstrated in Fig. 3. We stress that the total region of proton interactions keeps the Gaussian shape described by the first term in the right-hand side of Eq. (13). The Gaussian shape is preserved for the elastic profile as well but with the twice steeper exponent (see Eqs (18), (13)).

What concerns the longitudinal distances, it is commonly believed according to the parton model that they are much larger than the transverse size, especially for soft partons. Then the region of inelastic interactions would remind just a toroid, i.e. a tube, at the center of which only elastically scattered protons fly.

The plateau of $G(b)$ at small $b$ was confirmed in [15] as shown in Fig. 4. However, an additional substructure at the level $10^{-4}$ in a single point has been mentioned there. The function $G_{\text {inel }}=0.25 G$ at $7 \mathrm{TeV}$, plotted there, slightly decreases at $b=0$ compared to its values at $b=0.1-0.3 \mathrm{fm}$ (compare the numerical values shown in the Figure). This could be an indication that $Z$ becomes somewhat less than 1 already at $7 \mathrm{TeV}$ and the transition to the concave shape starts at this energy. At the same time, no decrease is seen in Fig. 2. This disagreement is especially surprising because the same model [12] was used in papers [11, 15] to fit experimental results at $7 \mathrm{TeV}$. However, we see that the excess at impact parameters $0.1-0.3 \mathrm{fm}$ compared to the center pointed out in [15] reveals itself in the fourth digits only while the error bars of the slope $B$ and the total cross section $\sigma_{t}$ are larger by an order of magnitude. This excess is so small that it can be explained either by insufficient precision in determining the values of $B$ and $\sigma_{t}$ (and, consequently, of $Z$ ) and also by inaccuracy in account for different scales or by different procedures adopted in the papers [11, 15] for extrapolations to the ranges of transferred momenta where there are no experimental data yet.

Thus it seems too early to make any (even preliminary) statements. However, the comparison of the results of [11] and [15] shows that we are in the critical regime of elastic scattering with $Z \approx 1$ at $7 \mathrm{TeV}$ as was pointed out in [14. Let us stress that at good precision of experimental data the proposed approach allows to analyse the fine structure of the core of the interaction region at the very small scales in distinction to the less precise correlation 
methods. Therefore we should pay special attention to evolution of the parameter $Z$ at higher energy of $13 \mathrm{TeV}$ which will become available soon. The especially precise measurements of the diffraction cone slope $B$ and the total cross section $\sigma_{t}$ would be very desirable.

Another consequence of Eq. (14) follows from study of energy evolution of $G(s, 0)$ shown in the Table. In connection with the torus-like concave structure, it is interesting to point out the value of $Z=0.64$ or $G(s, 0)=0.68$ at $\sqrt{s}=2.70 \mathrm{GeV}$ and maximum 1 at $b_{m}^{2}=4 B \ln 2$. However, at this rather low energy the whole analysis should be redone with account of the diffraction cone behavior, total cross section and the value of the ratio $\rho$. One also notices that, in the energy interval $4 \mathrm{GeV}<\sqrt{s}<8 \mathrm{GeV}$, the values of $Z$ are slightly larger than 1 so that the values of $G(s, 0)$ are smaller but very close to 1. These facts ask for further studies in the energy interval $2.7 \mathrm{GeV}<\sqrt{s}<8$ $\mathrm{GeV}$ especially in view of proposed experiments in Protvino. The dark core must be smaller at lower energies than at LHC because of smaller values of $B$. Moreover, the contribution due to the real part of the amplitude is larger at these energies as well as larger $|t|$ beyond the diffraction cone can be important. The dependence of $Z$ on energy shown in the Table looks as if the interaction region at low energies becomes black at the center $b=0$ but at higher energies up to ISR loses this property trying to restore it at the LHC.

In principle, the positivity of the inelastic cross section

$$
\sigma_{i n}=\frac{\pi B}{Z^{2}}(4 Z-1) \geq 0
$$

admits the value of $Z$ as small as 0.25 which asymptotically corresponds to $\sigma_{e l}=\sigma_{t}$ and $\sigma_{i n}=0$. The values of $Z<0.5$ lead to negative values of $\sigma_{\text {inel }}(s, b)$, i.e. to negative Fourier - Bessel transforms of $g(p, \theta)$ in Eq. (5). They are not forbidden if the relative phases of matrix elements of inelastic processes $M_{i}$ in Eq. (5) interfere in such a way. Unfortunately, we have no knowledge about them. This possibility was treated as another branch of the solution of the unitarity condition and named as antishadowing or refractive scattering in [16] and as resonant disk modes in [17]. However, the value of $Z$ is close to 1 even at LHC energies, and this regime asking for $Z<0.5$ is, surely, shifted to extremely high energies if it can be observed at all. The approach to asymptotics is argued as following the logarithmic dependences of cross sections $\sigma_{t} \propto \sigma_{e l} \propto \ln ^{2} s$ and $\sigma_{i n} \propto \ln s$. The depletion of $G(s, 0)$ in [15] was ascribed to this regime by mistake since the values of $Z$ are near 1 
at $7 \mathrm{TeV}$ but not as small as 0.5 .

\section{New tendencies of inelastic collisions}

The maximum absorption at central collisions at LHC energies must reveal itself in some special features of inelastic collisions in such a critical regime with $Z \approx 1$. The diffraction cone contributes mostly to $G(s, b)$. The large- $|t|$ elastic scattering can not serve as an effective trigger of the black core. One of the typical features of the high energy inelastic processes is the production of high energy jets, i.e. collimated groups of particles. The inelastic exclusive processes can be more effectively used for the analysis of the central black core. One needs such triggers which enhance its contribution. Following the suggestions of [10, 18], it becomes possible [13] to study the details of the central core using the experimental data of CMS collaboration at $7 \mathrm{TeV}$ about inelastic collisions with high multiplicity triggered by the hadron jet production [19] as well as some other related data. Triggers (charged particles or jets) with large transverse momenta are produced in central collisions. Therefore, the black plateau in the central part of the interaction region with $b<0.4-0.5 \mathrm{fm}$ should result in the corresponding plateau of the charged particle density in the transverse region $60^{\circ}<|\Delta \phi|<120^{\circ}$ defined as follows

$$
\mu_{t r}=\frac{N_{c h}^{t r}}{\Delta \eta \Delta(\Delta \phi)},
$$

where $N_{c h}^{t r}$ is the charged particle multiplicity in the transverse region, $\Delta \eta$ is the pseudorapidity range studied, $\Delta(\Delta \phi)$ is the azimuthal width of the transverse region. This is really the case as shown in Fig. 5.

Let us explain it. Starting from large transverse momenta of triggers on the righthand side of Fig. 5 and going to the left, we somehow feel at the beginning the central region from $b=0$ to the end of the plateau. Then the density of accompanying particles in the transverse region should not change until we approach the end of it. Only then the decrease of the distribution of the accompanying particles should start. The difference in positions of such a decrease in the two plots is defined by the difference in the choice of the leading trigger used by the two collaborations. The flat dependence of $\mu_{t r}$ on $p_{\mathrm{T}}$ shows that activity in the transverse region is independent of hard process scale, provided that scale is hard enough that all proton-proton interactions are central. 


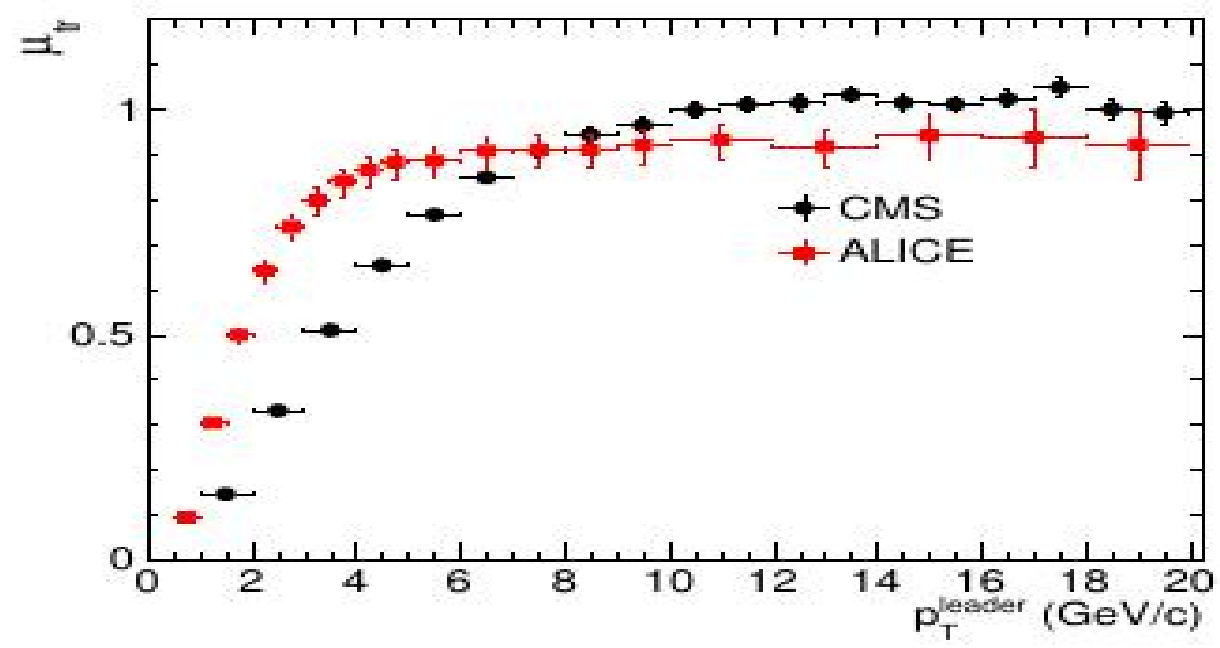

Fig. 5. Charged-particle density in the transverse region as a function of $p_{\mathrm{T}}$ of leading object [13] (CMS - charged-particle jet, ALICE - charged particle). CMS analyses particles with $p_{\mathrm{T}}>0.5 \mathrm{GeV} / c$ and $|\eta|<2.4$, ALICE - with $p_{\mathrm{T}}>0.5 \mathrm{GeV} / c$ and $|\eta|<0.8$.

Many other characteristics of such inelastic processes considered in [13] support this conclusion. Usage of very high multiplicity events in combination with jet properties is crucial. In particular, the most important observation is that the significant reduction of jet rate at very high multiplicities compared to MC predictions asks for new inputs in the models. Separating the core contribution with the help of these triggers, one comes to the important conclusion that the simple increase of the geometrical overlap area of the colliding protons does not account for properties of jet production at very high multiplicities. It looks as if the parton (gluon) density must strongly increase in central collisions and rare configurations (fluctuations) of the partonic structure of protons are involved. Up to now, the paper [13] is the first attempt to explain the distinctive features of jets and underlying event properties at LHC energies. The correlation studies of jets (see, e.g., [20]) can be used for further femtoscopy of the fine structured system. Surely, some further proposals to learn the critical regime at $7-8 \mathrm{TeV}$ will be proposed.

At the same time, implications of energy evolution of $Z$ (if it will be 
observed!) for inelastic processes are of great interest. The relative roles of elastic and inelastic cross sections will start approaching one another in the case if values of $Z$ would diminish below 1 with increase of energy. The mean multiplicity will, probably, decrease because of the more peripheral origin of newly created particles. The decreasing role of central interactions can lead to some changes in the shape of the multiplicity distributions (lower tails?) and diminished share of jets which will acquire new features. Jets will become produced at the periphery in distinction to the situation described above. That would imply that they will have to penetrate larger distances in transverse direction compared to forward directions. It would give rise to their stronger depletion in the transverse plane and, therefore, to the azimuthal asymmetries which were looked for in [20. Surely, there will be found other criteria of transition to the concave shape of the interaction region of inelastic processes.

Hardly, we will be able to reach the regime with extremely small values of $Z<0.5$ when inelastic processes would play negigibly small role compared to elastic ones.

\section{Elastic scattering outside the diffraction cone}

This problem attracts special attention since 60th when the experimental data about the differential cross section at high energies and rather large transferred momenta appeared for the first time. It was observed that the exponential $t$-regime in the diffraction cone is replaced by the exponential $\sqrt{t}$-regime at larger angles. The latter region of angles was called the Orear region in the name of its investigator.

The unitarity condition happened to be very successful in that region of angles as well. Theoretically, it can be approached considering the unitarity condition (3) directly in $s, t$-variables without using the Fourier - Bessel transform as it was done at small angles. It was shown a long time ago [5, 21] that the imaginary part of the amplitude $f$ outside the diffraction cone can be derived from the general unitarity condition (3) which is reduced there to the inhomogeneous linear integral equation

$$
\operatorname{Im} f(p, \theta)=\frac{p \sigma_{t}}{4 \pi \sqrt{2 \pi B}} \int_{-\infty}^{+\infty} d \theta_{1} \exp \left(-B p^{2}\left(\theta-\theta_{1}\right)^{2} / 2\right) r_{\rho} \operatorname{Im} f\left(p, \theta_{1}\right)+g(p, \theta)
$$


where $r_{\rho}=1+\rho(s, 0) \rho\left(s, \theta_{1}\right)$. This reduction becomes possible because the contribution from asymmetrical configuration of scattering angles in the first term of Eq. (3) dominates due to the steep Gaussian fall-off inside the diffraction cone. Because of the sharp fall off of the amplitude with angle, the principal contribution to the integral arises from a narrow region around the line $\theta_{1}+\theta_{2} \approx \theta$. Therefore one of the amplitudes should be inserted at small angles within the cone as a Gaussian while another one is kept at angles outside it.

It can be solved analytically (for more details see [5, 21]) with two assumptions that the role of the overlap function $g(p, \theta)$ is negligible outside the diffraction cone and the function $r_{\rho}$ may be approximated by a constant, i.e. $\rho\left(\theta_{1}\right)=\rho_{l}=$ const.

Let us assume that the overlap function is negligible at these transferred momenta ${ }^{1}$. Then, the eigensolution of the homogeneous linear integral equation is

$\operatorname{Im} f(p, \theta)=C_{0} \exp \left(-\sqrt{2 B \ln \frac{Z}{r_{\rho}}} p \theta\right)+\sum_{n=1}^{\infty} C_{n} \exp \left(-\left(\operatorname{Re} b_{n}\right) p \theta\right) \cos \left(\left|\operatorname{Im} b_{n}\right| p \theta-\phi_{n}\right)$

with

$$
b_{n} \approx \sqrt{2 \pi B|n|}(1+i \operatorname{sign} n) \quad n= \pm 1, \pm 2, \ldots
$$

This expression contains the exponentially decreasing with $\theta$ (or $\sqrt{|t|}$ ) term (Orear regime!) with imposed on it oscillations much stronger damped by their own exponential factors $b_{n}$ compared to the first term. The critical role in the exponent of the first leading term which determines the rate of decrease of the differential cross section is again played by the widely used above parameter $Z$ (12). The oscillating terms become pronounced only at smaller angles and reveal themselves as the dip in the vicinity of the diffraction cone. The elastic scattering differential cross section outside the

\footnotetext{
${ }^{1}$ The assumption of smallness of the overlap function outside the diffraction cone is appealing intuitively. The point is that particles newly created in high energy inelastic processes move mainly inside narrow angular cones along the directions of the primary hadrons. Therefore, the geometrical overlap of two narrow cones, whose axes are turned by the comparatively large angle $\theta$, is negligibly small. Moreover, it was shown to be valid [22, 23] by direct computation of the overlap function from experimental data in a wide energy interval up to LHC energies.
} 
diffraction cone (in the Orear regime region) is

$$
\begin{aligned}
\frac{d \sigma}{p_{1} d t} & =\left(e^{-\sqrt{2 B|t| \ln \frac{Z}{r_{\rho}}}}\right. \\
& \left.+p_{2} e^{-\sqrt{2 \pi B|t|}} \cos (\sqrt{2 \pi B|t|}-\phi)\right)^{2}
\end{aligned}
$$

with the parameters $p_{1}$ and $p_{2}$ tightly related to $C_{0}$ and $C_{1}$. Namely this formula was used in Refs [24, 23] for fits of experimental data about differential cross sections in a wide energy range. The ratio $\rho$ was approximated by its average values in and outside the diffraction cone so that $r_{\rho}=1+\rho_{0} \rho_{l}$ where $\rho_{l}$ is treated as the average value of $\rho$ in the Orear region.

The fits at comparatively low energies [24] are consistent with $r_{\rho} \approx 1$, i.e., with small values of $\rho_{l}$ close to zero. When $Z=1$, as it happens at $7 \mathrm{TeV}$ (see the Table above), the exponent of the main first term is very sensitive to the value of $\rho_{l}$ outside the diffraction cone. For the first time, that allowed to estimate the value of $\rho$ in the Orear region at $7 \mathrm{TeV}$ [23]. The great surprise of the fit of TOTEM data was necessity to use the large (in modulus) negative value of $\rho_{l} \approx-2.1$ if $\rho_{0}=0.14$ (as it was at ISR energies). Otherwise, the slope of the first term of Eq. (22) in the Orear region would be predicted to be equal to zero (constancy!) if $Z=1$ and $r_{\rho}=1$. It becomes larger in modulus $\rho_{l} \approx-3$ if the TOTEM value $\rho_{0}=0.1$ obtained at $7 \mathrm{TeV}$ is used. Moreover, these values of $\rho_{l}$ can be considered as upper limits because the effective value of $\rho_{0}$ inside the diffraction cone can be even smaller in view of its widely discussed zero there. No models have yet explained this finding. Further progress in solving the unitarity equation with proper dependence of $\rho$ in and outside the diffraction cone is needed.

The slope of the differential cross section in the Orear region becomes a very sensitive indicator of the mutual behavior of $Z$ and $\rho_{l}$. Possible decrease of the value of $Z$ with increasing energy and transition to the torus regime, discussed above, would ask for further evolution of the ratio $\rho$ to increasing in modulus negative values.

The predictive power of the solution (22) lies in its exponential behavior in $\sqrt{|t|}$ with quite definite analytically calculable exponent and imposed on it oscillations. Unfortunately, one can not definitely state where its bounds on the $t$-axis are and has to rely on accuracy of fits within some range of $t$. Nevertheless, as was mentioned above, some important estimates of the value of $\rho$ in the Orear region have been done. Another shortcoming of the of the solution $(22)$ is its ignorance of the energy dependence. Therefore, no 
predictions are made concerning higher energies. Not only the normalization coefficients $C_{0}, C_{n}$ are unknown but the exponent of the leading term suffers also from unpredictable energy behavior of $B, \sigma_{t}$ and $r_{\rho}$. Only with results on $B, \sigma_{t}$ obtained from experiment at higher energies it is possible to estimate the ratio $\rho$ in the Orear region assuming it to be constant in there.

In parallel, there exists a variety of phenomenological models with numerous adjustable parameters which were proposed in attempts to describe experimental data. The theoretical arguments in favour of them and their main features are reviewed in some detail in [6]. Most of them describe quite well (albeit with some precaution) the behavior of the differential cross section in the diffraction cone. Therefore, if applied to the problem, they would reproduce the main features of the shape of the interaction region discussed in the previous section. The imaginary part of the amplitude dominates in there. At the same time, they failed in their predictions at $7 \mathrm{TeV}$ in the Orear region as shown in Fig. 1(right). The main problem lies in their inability to predict the energy dependence of adjustable parameters. Only some qualitative guesses can be used.

Surely, it is much easier to use such guesses and to fit these parameters by the newly available data. That is what was successfully done a'posteriori, for example, in papers [11, 15, 25]. First two of them used the model [12] proposed a long ago, while a completely new form of the amplitude was analyzed in [25].

Independently of success or failure of these models in describing the Orear region, all of them have a common heuristic feature. To explain such prominent characteristics of the differential cross section as the dip (see Fig. 1 (right)), they have to assume that it originates at the transferred momentum $t$ where the imaginary part of the amplitude (dominating until then within the diffraction cone!) becomes equal to zero. The real part contributes in the dip to the differential cross section. The models differ in the numbers of zeros in real and imaginary parts and in their positions except the definitely fixed $t$-value at the dip.

As an example, I show in Fig. 6 the corresponding graphs obtained for the model [25]. The zero of the imaginary part at the dip position is marked as $Z_{I}$. It is its only zero. The real part possesses two zeros. One of them, $Z_{R}(1)$, lies within the diffraction cone. This zero is typical for many models and was somehow envisaged in papers [26, 27] even though without definite predictions about its position. Its appearence leads to necessity to diminish the theoretical estimates of the average value of $\rho$ inside the cone which 


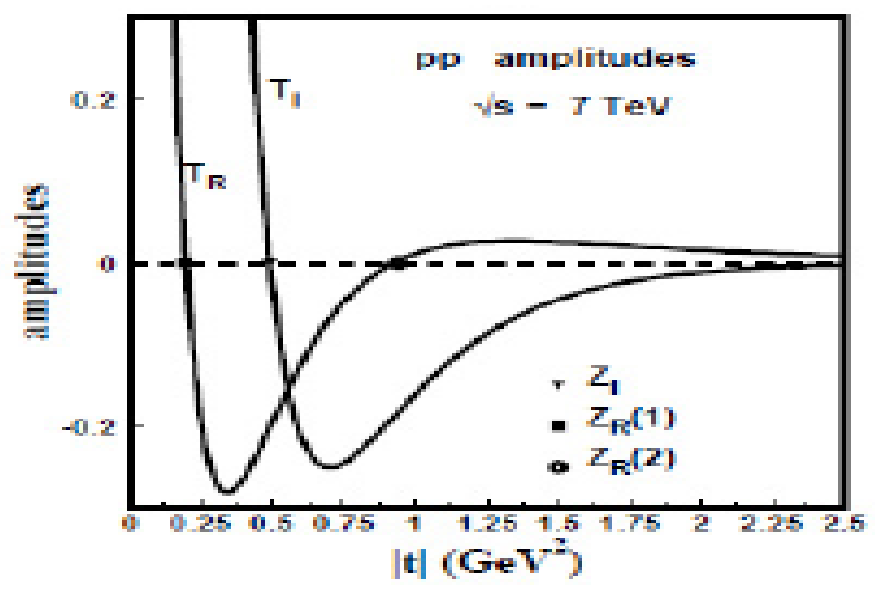

Fig. 6. Real $T_{R}=\operatorname{Re} f$ and imaginary $T_{I}=\operatorname{Im} f$ parts of the proton-proton amplitude at $7 \mathrm{TeV}$ according to a particular phenomenological model [25].

enters in the soluion (22) as $\rho_{0}$ but should be treated, strictly speaking, as the average value of $\rho$ inside the cone. In its turn, that would lead to larger (in modulus) values of $\rho_{l}$ as discussed above. The peculiar feature of the model [25] is that the real part crosses the abscissa axis and possesses the second zero $Z_{R}(2)$. Therefore, the ratio $\rho$ becomes negative in the Orear region albeit not large (in modulus) enough to correspond to estimates done in the unitarity approach. Anyway, there is some correspondence between them at the qualitative level. Other models have usually single zeros of real and imaginary parts and can not get the negative values of $\rho$ in the Orear region. Probably, this is the origin of their failure in predicting the LHC results.

It is worthwhile here to stress that no zeros of the imaginary part are predicted in the unitarity approach. The dip is explained [23] as the contribution of the oscillatory terms in (22) whose role increases at smaller transferred momenta. At the same time, one should critically remark that fits of the differential cross section in the Orear region with the help of the imaginary part of the amplitude only becomes selfcontradictory after the conclusion about the large value of $\rho_{l}$ in there is obtained. No consensus has been reached yet between these two approaches. In general, one can state that until now the $t$-dependence of real and imaginary parts is not understood theoretically. 


\section{Conclusions}

The usage of the indubitable general principle - the unitarity condition in combination with experimental results about the elastic scattering in the diffraction cone makes it possible to reveal the space image of the interaction region of protons and its evolution with energy as well as to estimate for the first time the average value of the real part of the elastic scattering amplitude beyond the diffraction cone.

The behavior of the real and imaginary parts of the elastic scattering amplitude as functions of energy $s$ and transferred momentum $t$ completely defines the properties of this process and, in some way, influences the properties of inelastic processes through the unitarity requirement. Our knowledge of this behavior is still quite limited. QCD methods do not work. The general QCD statements and phenomenological models are usually considered.

It is shown that the geometrical space shape of the interaction region of protons is mainly determined by their elastic scattering at small angles. The absorption at its center is determined by a single energy-dependent parameter $Z$. The region of full absorption extends to quite large impact parameters up to $0.5 \mathrm{fm}$ if $Z$ tends to 1 . This happens at the LHC energy $\sqrt{s}=7 \mathrm{TeV}$ where the critical two-scale (the black central core and the more transparent peripheral region) structure of the interaction region of protons becomes well pronounced. The sharp separation of these two regions leads to special consequences both for elastic and inelastic processes. The behavior of the parameter $Z$ at higher energies is especially important for the evolution of the geometry of the interaction region. The assumption about its further decrease at higher energies results in drastic change of the geometry predicting the tendency for evolution to the completely unexpected toroid (tube)-like (or ring-like in two dimensions) configuration, where the core becomes absolutely penetrable, and the complete absorption region is shifted to some finite impact parameter.

The value of $Z=1$ attained at the LHC energies is also crucial for the behavior of the elastic scattering differential cross section outside the diffraction cone. In this case, its slope there becomes fully defined by the ratio of the real part of the amplitude to its imaginary part which is yet unknown at LHC energies in this range of the transferred momenta. No ways to its direct measurement is foreseen nowadays. Therefore, it is very important that the analysis of experimental data at $7 \mathrm{TeV}$ about the slope of the differential cross section inside the Orear region with the help of the 
unitarity condition provides the estimate of its average value and reveals that this ratio is negative and surprisingly large in modulus. The predictions of phenomenological models are contradictory in this region of transferred momenta. In general, these models should be checked for their selfconsistency by calculation of the overlap functions $g(p, \theta)$ for each of them. This is possible because the integral in the unitarity relation (3) can be computed with both real and imaginary parts of the amplitude for a particular model known. The measurement of the rate of decrease of the differential cross section in the Orear region becomes very important at higher energies because it happens to be very sensitive to the mutual behavior of $Z$ and $\rho_{l}$ with increase of energy.

Thus, the unitarity condition provides us many inspiring guides about hadron interactions which should be taken into account by other approaches.

I am grateful for support by the RFBR grants 12-02-91504-CERN-a, 1402-00099 and the RAS-CERN program.

\section{References}

[1] Antchev G et al. (TOTEM Collab.) Europhys. Lett. 9541001 (2011)

[2] Antchev G et al. (TOTEM Collab.) Europhys. Lett. 9621002 (2011)

[3] Antipov Yu M et al. Phys. Rev. Lett. 351406 (1975)

[4] Zotov N P, Rusakov S V, Tsarev V A Elem Part and Nucl 111160 (1980)

[5] Andreev I V, Dremin I M ZhETF Pis'ma 6810 (1967)

[6] Dremin I M Physics-Uspekhi 563 (2013)

[7] Van Hove L Nuovo Cimento 28798 (1963)

[8] Kraus E, Mesick K E, White A, Gilman R, Strauch S, arXiv:1405.4735

[9] Sinyukov Yu M, Shapoval V M Phys. Rev. D 87094024 (2013)

[10] Frankfurt L, Strikman M, Weiss C Phys. Rev. D 83054012 (2004)

[11] Dremin I M, Nechitailo V A Nucl. Phys. A 916241 (2013) 
[12] Amaldi U, Schubert K R Nucl. Phys. B 166301 (1980)

[13] Azarkin M Yu, Dremin I M, Strikman M Phys. Lett. B 735244 (2014)

[14] Dremin I M JETP Lett. 99243 (2014)

[15] Alkin A, Martynov E, Kovalenko O, Troshin S M Phys. Rev. D 89 091501(R) (2014)

[16] Troshin S M, Tyurin N E Phys. Lett. B 316175 (1993)

[17] Anisovich V V, Nikonov V A, Nyiri J, arXiv:1408.0692

[18] Frankfurt L, Strikman M, Weiss C Phys. Rev. D 69114010 (2004)

[19] CMS Collaboration Eur. Phys. J. C 732674 (2013)

[20] CMS Collaboration PAS FSQ-13-005 (2013)

[21] Andreev I V, Dremin I M Sov. J. Nucl. Phys. 8473 (1969)

[22] Andreev I V, Dremin I M, Steinberg D N Sov. J. Nucl. Phys. 11261 (1970)

[23] Dremin I M, Nechitailo V A Phys. Rev. D 85074009 (2012)

[24] Andreev I V, Dremin I M, Gramenitskii I M Nucl. Phys. 10137 (1969)

[25] Kohara A K, Ferreira E, Kodama T Eur. Phys. J. C 732326 (2013); arXiv:1408.1599

[26] Martin A Lett. Nuovo Cim. 7811 (1973)

[27] Martin A Phys. Lett. B 404137 (1997) 